\title{
Oral mucositis - significant complication of pediatric patients undergoing hematopoietic stem cell transplantation
}

\author{
Oana Otilia Niculita ${ }^{1,2}$, Andra Daniela Marcu ${ }^{1,2}$, Ana Maria Bica ${ }^{2}$, \\ Roxana Elena Doncu', Anca Colita ${ }^{1,2}$, Cristina Georgiana Jercan ${ }^{1,2}$ \\ 1 "Carol Davila" University of Medicine and Pharmacy, Bucharest, Romania \\ ${ }^{2}$ Fundeni Clinical Institute, Bucharest, Romania
}

\begin{abstract}
Introduction. The prognosis of pediatric solid tumors has improved in recent years through the use of multimodal treatment - chemotherapy, local control (surgery and radiotherapy) and hematopoietic stem cell transplantation (HSCT) for high-risk forms. A limiting factor of these transplant procedures is short-term toxicity, oral mucositis (OM) being associated with potential severe evolution. The introduction of tandem stem cell transplant procedures, which increase the intensity of chemotherapy treatment and thus increase the response rate, is associated with increased toxicity. In this study we evaluated the impact of multiple transplant procedures on the severity and evolution of OM. Materials and methods. This retrospective study included 57 pediatric patients diagnosed with solid tumors (neuroblastoma, germ cell tumor, Ewing sarcoma, nephroblastoma) who underwent HSCT in the Pediatric Bone Marrow Transplantation Unit of Fundeni Clinical Institute, between January 2002 and December 2020. Patients were divided into 2 study groups: the first group included patients who had one HSCT procedure (43 patients) and the second group included patients who benefited from multiple HSCT procedures (14 patients). We evaluated the degree and average extent of $\mathrm{OM}$, associated risk factors, duration of analgesic treatment and parenteral nutrition, as well as infectious risk. Conditioning regimens were based on administration of busulfan, thiotepa or ifosfamide. All patients received prophylactic antimicrobial therapy and followed a daily oral care protocol.

Results. The 57 patients underwent 73 HSCT procedures for the following diagnoses: 36 neuroblastoma, 6 germinal cell tumor, 12 Ewing sarcoma and 3 Wilms tumor. Patients were diagnosed, staged and treated according to international protocols. The study highlights the influence on the degree of OM: delayed platelet engraftment and increased parenteral nutrition requirements correlated with the number of HSCT procedures per patient. Infectious risk was higher for patients from the second study group.

Conclusions. OM treatment remains prevention-centered and identifying effective measures, encouraging modern treatment strategies and developing practical evidence-based guidelines are imperative.
\end{abstract}

Keywords: solid tumors, hematopoietic stem cell transplantation, oral mucositis, children

\section{INTRODUCTION}

Solid tumors account for about $30 \%$ of all pediatric cancers, with a low survival rate for patients in high-risk group. Increasingly common indication, hematopoietic stem cell transplantation (HSCT) represents the infusion of multipotent stem cells obtained from the patient (autologous transplant) or donor (allogeneic transplant). This complex procedure is part of the aggressive solid tumor's management protocol, along with surgery, chemotherapy, radiotherapy and/or immunotherapy.

The encouraging response associated with autologous transplantation led to the hypothesis that in- creased intensity chemotherapy could be associated with better remission rate. Thus, study protocols have emerged to evaluate response after double or triple transplant procedures, but with redoubtable toxic effects that lead to rapid decline of nutritional status and potentially severe progression [1,2].

Mucositis, the most common complication of pediatric patients undergoing HSCT, involves inflammatory-ulcerative lesions of the entire digestive tract, having multiple etiologies: infections, immune deficits, intensive drugs/chemotherapy or body irradiation $[3,4]$. Mucositis incidence is $40-80 \%$ and depends on the intensity of cytotoxic regime or use of total body irradiation. Although it is not an infectious 
disease, it negatively influences the healing process by developing secondary digestive colonization [5]. In context of severe neutropenia secondary to conditioning regimen, patients become more susceptible to infections, including fungal or viral (in particular, herpetic). Developing oral mucositis (OM) allows bacteria from commensal flora to become pathogenic and determine bacteremia (e.g., streptococcus viridans) [6].

The oral mucosa has a protective function against toxic, mechanical or infectious agents and accelerates tissue recovery through rapid cellular turn-over. In addition, saliva has benefic antimicrobial and regenerative effects by modulating angiogenesis and preventing tissue dehydration [7]. Signs and symptoms of OM include erythema, edema, burning sensation, exaggerated thermal sensitivity, whitish patches throughout the oral cavity, bleeding, severe oral pain, speaking difficulties, feeding problems and weight loss [8]. Chemotherapy has both a direct effect on mucosal tissue and a secondary one due to myelosuppression $[9,10]$. The first stage is inflammatory/vascular where the mucosa releases cytokines that cause local tissue destruction. The second stage begins with the decrease of cellular turn-over, leading to atrophy or ulceration. These represent the evolution of the first 5 days of OM. The 3 rd stage means perforation of the epithelial tissue and formation of the actual ulcer, often colonized by gram positive and negative bacteria from the oral cavity. Products of bacterial metabolism amplify tissue destruction and stimulate additional release of cytokines promoting inflammation. The last stage is characterized by tissue restoration, initiated in the submucosa, after about 12-16 days [11].

\section{PURPOSE}

The study evaluates how the number of HSCT procedures performed on patients with solid tumors influences OM degree and the impact it has on clinical evolution.

\section{MATERIALS AND METHODS}

We conducted an observational retrospective study that evaluated the severity and evolution of $\mathrm{OM}$ in pediatric patients diagnosed with solid tumors who underwent a single HSCT procedure compared to those who had multiple procedures in the Pediatric Bone Marrow Transplantation Unit of Fundeni Clinical Institute between January 2002 and December 2020. The inclusion criteria targeted patients between 0 and 18 years old who had at least one HSCT proce- dure for the following diagnoses: neuroblastoma (NBL), germinal cell tumor (GCT), Ewing sarcoma (ES) or Wilms tumor (WT), after their caregivers signed informed consent. Exclusion criteria are the following: patients out of age range, other oncological diagnoses, other types of HSCT procedures, legal guardians who refused to sign informed consent.

\section{Population and study procedures}

Patients were divided into 2 study groups: the first group included patients who had one HSCT procedure (43 patients) and the second group included patients who benefited from multiple HSCT procedures (14 patients). Patients received different conditioning regimens, depending on the diagnosis and type of procedure (single or multiple), listed in Table 1.

\section{TABLE 1. Conditioning regimes}

\begin{tabular}{|l|l|l|l|}
\hline Diagnosis & HSCT 1 & HSCT 2 & HSCT 3 \\
\hline NBL & $\begin{array}{l}\text { Bu-Mel } \\
\text { CEM }\end{array}$ & $\begin{array}{l}\text { Thiotepa- } \\
\text { Cyclophosphamide } \\
\text { Bu-Mel } \\
\text { TMF }\end{array}$ & \\
\hline ES & Bu-Mel & $\begin{array}{l}\text { Melphalan- } \\
\text { Etoposide }\end{array}$ & \\
\hline GCT & $\begin{array}{l}\text { Paclitaxel- } \\
\text { Ifosfamide }\end{array}$ & $\begin{array}{l}\text { CET } \\
\text { Paclitaxel- } \\
\text { Ifosfamide }\end{array}$ & $\begin{array}{l}\text { CET } \\
\text { ICE } \\
\text { ICE }\end{array}$ \\
\hline WT & Paclitaxel-Thiotepa & \\
\hline
\end{tabular}

Bu-Mel: busulfan-melphalan; CEM: carboplatin-etoposide-melphalan; TMF: thiotepa-melphalan-fludarabine; CET: carboplatin-etoposide-thiotepa; ICE: ifosfamide-carboplatin-etoposide

Patients performed daily oral care protocol (OCP) starting from the first day of the conditioning regime to OM resolution: mouthwashes with sodium bicarbonate solutions, antiseptic solutions with chlorhexidine spray and/or oral gel containing bioactive combination of zinc, taurine and polyvinylpyrrolidone (PVP) gluconate according to standard protocol. OCP was displayed in each room for the patients and their caregivers to follow, and nurses were trained to implement it and collect data. Compliance with OCP was assessed by checking patient journal data and evaluating oral solution consumption. Patients who received melphalan-conditioning were advised to use ice chips starting 5 minutes before chemotherapy and throughout the infusion (60 minutes). For infants and toddlers, iced antiseptic lollipops were used to achieve vasoconstriction in oral mucosa.

Medical personnel were trained to assess daily OM score in accordance with WHO recommendations, as shown in Table 2. Painkiller drugs were administered starting with grade 2 of OM, with progressively increased tramadol infusion. Absence of 
therapeutic response led to using patches of fentanyl or intravenous morphine. Parenteral nutrition was introduced starting with grade $3 \mathrm{MO}$.

TABLE 2. The severity of OM (according to WHO) [12]

\begin{tabular}{|l|l|}
\hline Severity grade of OM (according to WHO) \\
\hline Grade $\mathbf{0}$ & No changes \\
\hline Grade $\mathbf{1}$ & Mucosal hyperemia \\
\hline Grade $\mathbf{2}$ & $\begin{array}{l}\text { Hyperemia + ulcerative lesions + patient tolerates } \\
\text { solid nutrition }\end{array}$ \\
\hline Grade $\mathbf{3}$ & $\begin{array}{l}\text { Extensive ulcerative lesions + patient DOES NOT } \\
\text { swallow solid foods }\end{array}$ \\
\hline Grade 4 & $\begin{array}{l}\text { Feeding is IMPOSSIBLE + extensive ulceration + } \\
\text { hypersalivation }\end{array}$ \\
\hline
\end{tabular}

Infectious colonization was evaluated through weekly samples: nasal and pharyngeal swab, ear swab, urine and stool specimens/rectal swab. All patients received prophylactic antibiotics, antiviral and antifungal treatment according to standard protocols, and granulocyte growth factor after transplantation. Blood cultures were taken from all febrile patients, both from peripheral blood and central venous catheter (CVC). In case of fever or confirmed infection, the number of antifungal and antibacterial treatment lines was recorded. Antimicrobial therapy has been progressively escalated according to standard protocols. The first antibiotic line for neutropenic patients, often given empirically, was intravenous piperacillin/ tazobactam or cefepime. Treatment escalation includes adding vancomycin or linezolid if CVC infection is detected; metronidazole, colistin or ceftazidime/avibactam in abdominal infections etc. Depending on clinical evolution and whether a certain germ was identified, treatment was given according to antibiogram. Initiation of iv antifungal therapy was recommended for deeply neutropenic patients with prolonged fever or for confirmed fungal infections. The duration of antimicrobial treatment is determined by the negative cultures or remission of fever, the therapy being continued until the patient has 3 days without fever.

During hospital admission, the following data was recorded: age, sex, type of conditioning, patient weight, temperature, feeding capacity, pain score according to the visual scale (VAS), degree of OM, number and appearance of stools, bacterial colonization, administration of painkillers and parenteral nutrition. From the patient's medical records, the blood count values were taken.

\section{Ethical considerations}

This study involves human subjects and receives the approval of the Ethics Committee of Fundeni
Clinical Institute no 11188/26.02.2021. The caregivers signed informed consent.

\section{Statistical processing methods}

The retrospective study was conducted through the analysis of patient medical records and observation sheets, the data being collected and processed statistically through Microsoft Office Excel 2019 and SPSS Statistics 24. For statistical analysis, we performed non-parametric tests (chi-square and MannWhitney U) expressed by median and rank, with statistically significant $\mathrm{p}$ value $<0.05$.

\section{RESULTS}

This study includes 57 patients, divided into 2 study groups: 43 underwent single HSCT procedure, 14 had multiple HSCT procedures. The sex ratio was $\mathrm{M}: \mathrm{F}=1.5: 1$, with median age of 5 years for children aged between 1 and 18 years old. 72 autologus transplantation procedures and 1 haploidentical HSCT were performed in 57 pediatric patients with the following diagnoses: NBL (36/57), GCT (6/57), ES $(12 / 57)$ and WT (3/57). Patients were diagnosed, staged and treated according to international protocols. The distribution by age group is shown in Figure 1.

Conditioning regimens (Figure 2a) were melphalan-based in 74\%, thiotepa-based for $9.5 \%$ and ifosfamide-based for $16.5 \%$ of patients. The most frequently used conditioning regimen was melphalan-based for the first procedure and thiotepabased for the second one (Figure $2 b$ ).

The patient distribution in each study group according to OM degree is represented in Figure 3. All patients developed OM, despite prophylaxis measures. A statistically significant influence of the number of transplant procedures on the severity of OM was found through chi-square test, resulting $\mathrm{p}<$ 0.001 , showing more severe OM for the second study group.

The evolution characteristics of patients belonging to the two study groups are shown in Table 3. Non-parametric tests were used for ordinal variables such as chi-square or Mann-Whitney U, establishing statistical significance for $\mathrm{p}<0.05$.

Routine evaluation of stool specimens showed digestive colonization in $73.3 \%$ of patients who underwent multiple HSCT procedures, revealing the increased frequency of simultaneous colonization with multiple infectious agents. The involved pathogens are shown in Figure 4. 


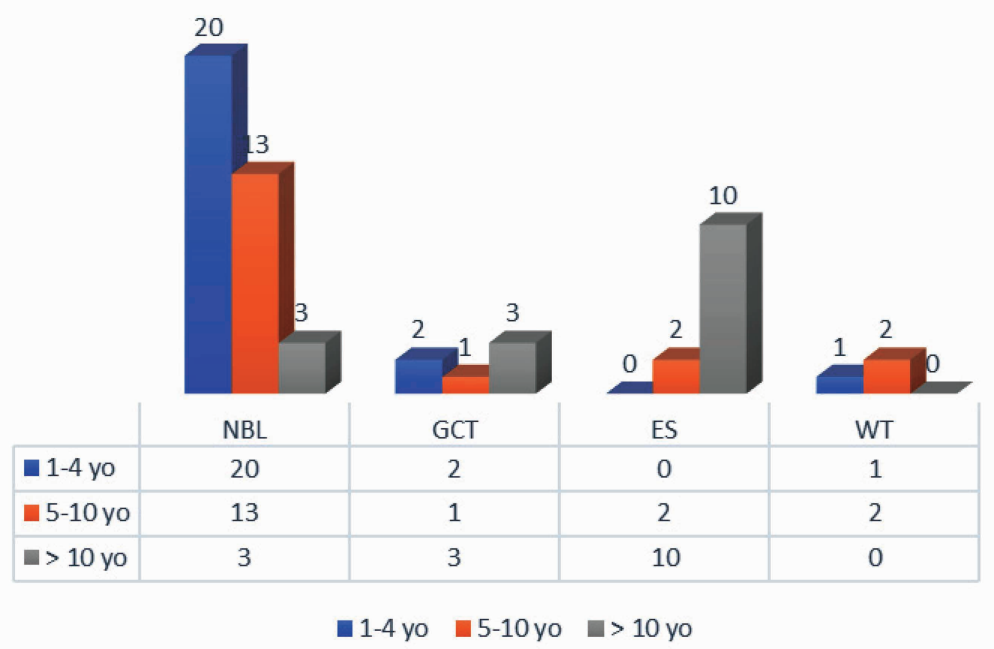

FIGURE 1. Case distribution by age and diagnosis

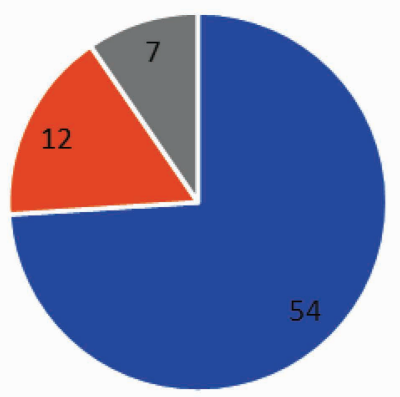

- Melphalan based

- thiotepa based

- ifosfamide -based

FIGURE 2A. Conditioning regimens according to chemotherapy agent - 73 procedures

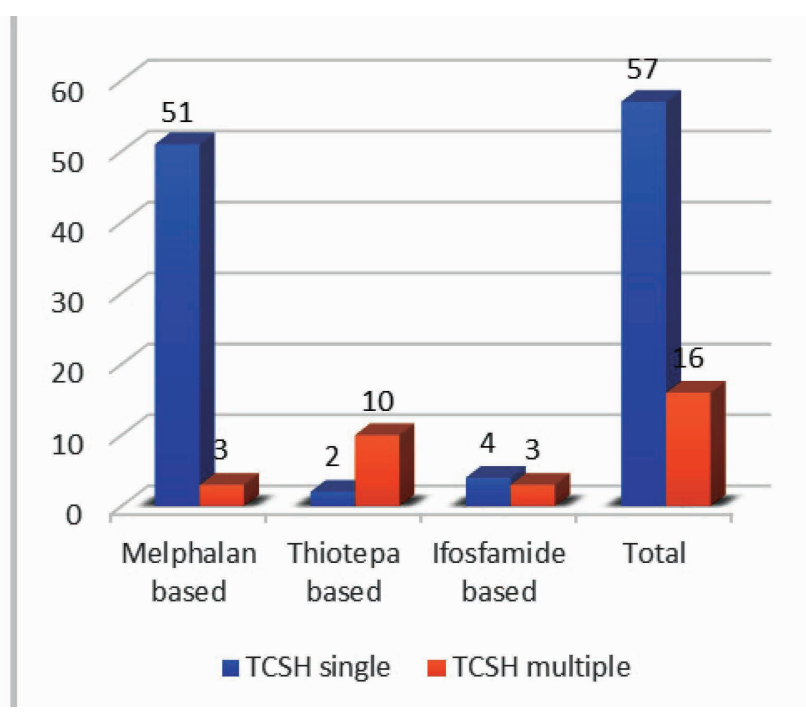

FIGURE 2B. Conditioning regimens distribution

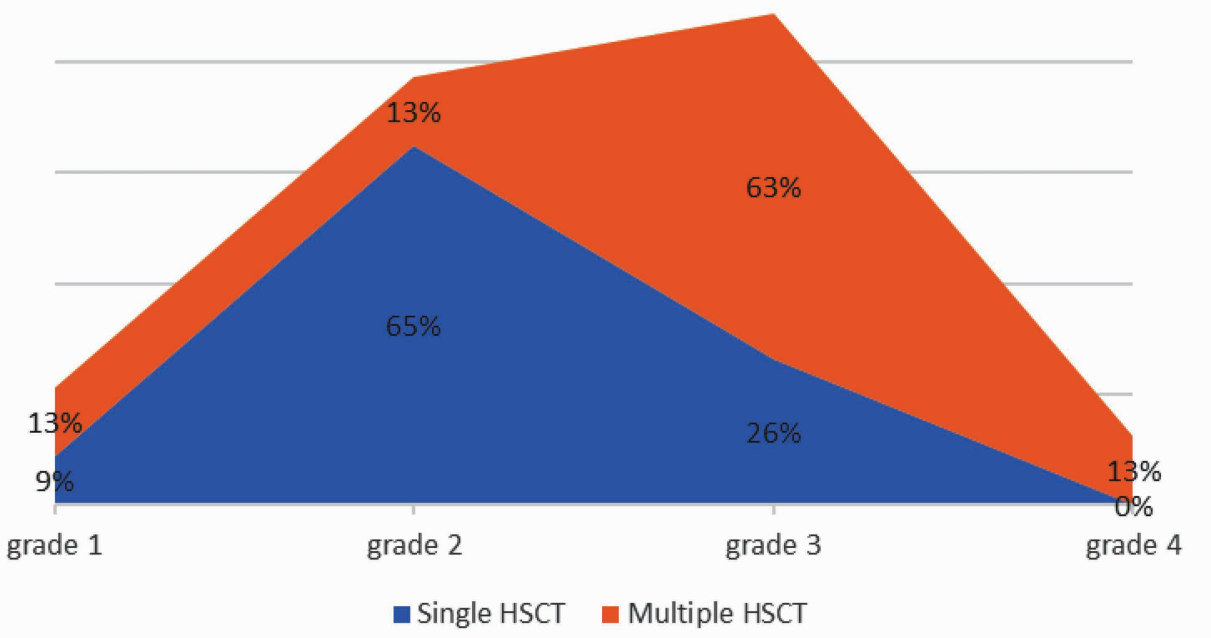

FIGURE 3. Degree of oral mucositis in the two study groups 
TABLE 3. Evolution characteristics

\begin{tabular}{|c|c|c|c|c|}
\hline & & $\begin{array}{l}\text { Single HSCT } \\
\text { (43 procedures) }\end{array}$ & \begin{tabular}{|l|} 
Multiple HSCT \\
(16 procedures) \\
\end{tabular} & P-value* \\
\hline \multirow[t]{2}{*}{ Engraftment } & Neutrophils & 14 days & 13 days & 0.243 \\
\hline & Thrombocytes & 13 days & 18 days & 0.029 \\
\hline & OM onset & Day +6 & Day 0 & $<0.001$ \\
\hline & OM duration & 9 days & 13 days & 0.068 \\
\hline & Opioid & $\begin{array}{l}7 \text { days } \\
\text { (9\% need morphine/fentanyl) }\end{array}$ & $\begin{array}{l}8 \text { days } \\
(10 \% \text { need morphine/fentanyl) }\end{array}$ & 0.521 \\
\hline & Parenteral nutrition & 9 days & 15 days & 0.030 \\
\hline & Hospitalization & 26 days & 29 days & 0.360 \\
\hline \multirow{2}{*}{$\begin{array}{l}\text { Intravenous fungal } \\
\text { treatment }\end{array}$} & 1 lines & $39 \%$ (16/41 pts) & $71.4 \%$ (10/14 pts) & \multirow[b]{2}{*}{0.193} \\
\hline & $\geq 2$ lines & 7.3\% (3/41 pts) & $0 \%(0 / 14$ pts $)$ & \\
\hline \multirow{3}{*}{$\begin{array}{l}\text { Intravenous } \\
\text { antibiotic } \\
\text { treatment }\end{array}$} & 1 iv line & $15.2 \%$ (7/46 pts) & $6.6 \%$ (1/15 pts) & \multirow{3}{*}{0.436} \\
\hline & 2 iv line & $54.3 \%$ (25/46 pts) & $40 \%$ (6/15 pts) & \\
\hline & $\geq 3$ iv line & $28.3 \%$ (13/46 pts) & $53.4 \%$ (8/15 pts) & \\
\hline
\end{tabular}

Fungal

Other Gram-negative

Clostridium difficile

Escherichia coli

Enterococcus

Positive
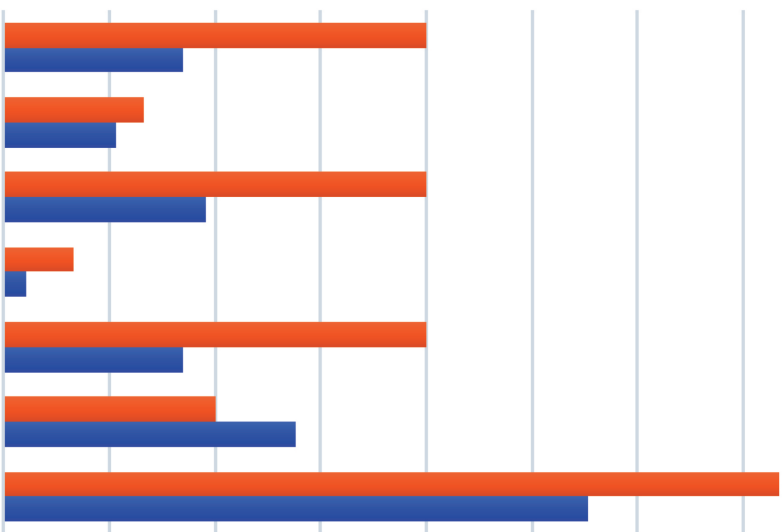

$0 \%$

$10 \% \quad 20 \% \quad 30 \%$

$40 \% \quad 50 \%$

$60 \%$

$70 \% \quad 80 \%$

Multiple HSCT $\quad$ Single HSCT

FIGURE 4. Digestive colonization after HSCT

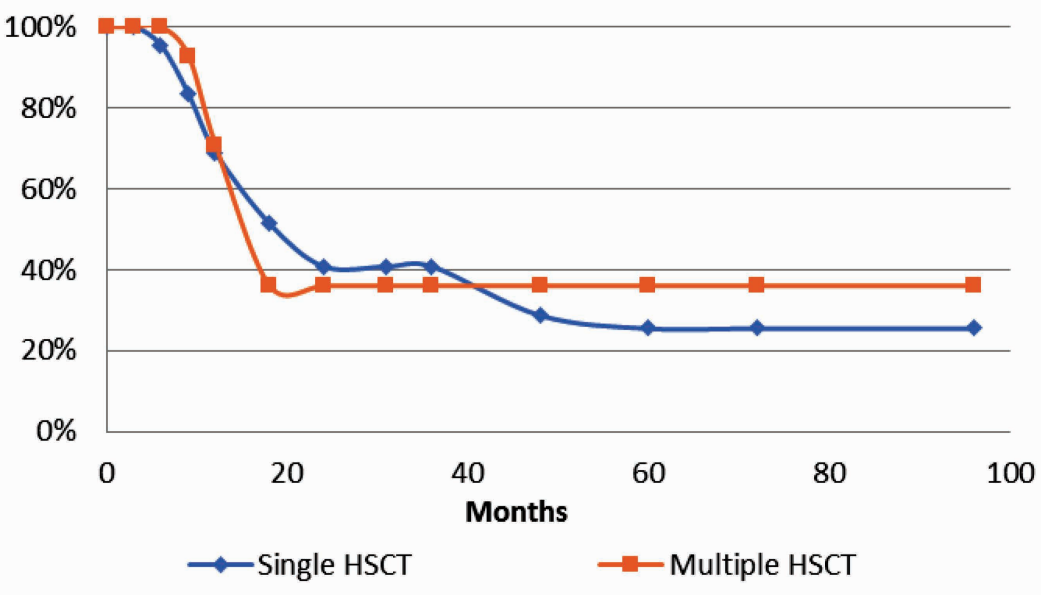

FIGURE 5. Overall survival for the 2 study groups

The overall survival was $26 \%$ for patients who underwent a single transplant procedure and $36 \%$ for those who had more than one procedure.

\section{DISCUSSION}

OM studies in pediatric patients undergoing HSCT procedures are reduced compared to adult 
population. Children metabolically differ from adults, continuing their growth and development process despite oncological treatment that lasts for years [13]. All patients in our study developed OM, although prophylaxis measures were taken.

The first step in OM prevention is rigorous oral hygiene and daily performance of oral care protocol (OCP). The management strategy includes mouthwash with saline solution - high safety profile, low toxicity, physiological properties, good vehicle; chlorhexidine, povidone iodine - well known antibacterial, prevent the formation of dental tartar (oral cavity reservoir for microorganisms); Sodium bicarbonate gentle and beneficial effect on the maintenance of oral hygiene. Therapeutic alternatives such as the one Banava et al. used involves fluoride from casein phosphopeptide - amorphous calcium phosphate enriched paste to stimulate salivary secretion, enhancing its protective effect [14]. Ariyawarhana et al. showed local benzidamin to be the only product with effective anti-inflammatory effect in OM prevention, through its inhibition of TNF $\alpha$ and IL-1 $\beta$ [15]. Often disapproved, oversaturated calcium-phosphate (Caphosol) acts as a lubricant with healing effect on oral mucosa, restores ionic balance and $\mathrm{pH}$ of saliva, modulates apoptosis, regulates mediators of inflammation [16].

Compliance with specific indications during conditioning regimens reduces toxicity of short half-life chemotherapy. Although controversial, cryotherapy is a universally valid strategy with no additional cost. $\mathrm{Lu}$ et al. demonstrated, on a group of 145 patients, the effectiveness of cryotherapy throughout conditioning regimen, but without clinical benefit if included in daily routine because it can cause mucous lesions [17]. In contrast, Kamsvåg et al. demonstrates its lack of effectiveness in a group of 49 pediatric patients performing HSCT [18]. Modern management of OM includes laser phototherapy for its anti-inflammatory, analgesic and regenerative effects. Furthermore, administration of keratinocyte growth factor is promoted, palifermin being recommended in MASCC/ISOO Study Group Protocol to be used 3 days before after HSCT [19,20].

In accordance with the higher degree of OM, our study shows statistically significant increase in parenteral nutrition requirements $(\mathrm{p}=0.030)$ in patients who have performed multiple HSCT procedures. Traditionally, nutritional support is taken into consideration for these patients and parenteral nutrition is administered starting with 3rd degree of OM. It is indicated in case of severe mucositis, intractable vomiting, severe malabsorption, prolonged diarrhea, or intestinal graft-versus-host disease after allogeneic
HSCT [21]. Total parenteral nutrition can however produce villous atrophy, with significant permeability of intestinal mucosa, alteration of microbiota and increased infectious risk [22-24] This explains the close link between immune suppression and infectious susceptibility due to intestinal barrier destruction. The Dutch team led by Kuiken conducted a national, subsequently international, observational study, based on an online questionnaire distributed in pediatric oncology centers, to identify nutrition strategies in OM after chemotherapy. The results highlighted the great variability of these strategies at both national and international level. Total parenteral nutrition, although leading to an acceptable nutritional status after chemotherapy, was correlated with increased frequency of infections, high antibiotic requirements and prolonged hospitalization. Their study also correlated the level of plasma citrulline $<10 \mu \mathrm{mol} / 1$ and increase in painkillers intake for patients who had severe mucositis and required total parenteral nutrition [25].

Some studies have shown that hypoalbuminemia and hypophosphatemia were common in patients with predominantly parenteral nutrition [26,27]. Hypoalbuminemia has multiple causes such as fluid redistribution, protein loss enteropathy or acute phase of infections. Their association with zinc depletion was correlated with the high number of positive blood cultures $[28,29]$. Delaying parenteral nutrition as much as possible and maintaining enteral nutrition seems logical and could support intestinal barrier function.

An important role is played by analgesic therapy usually initiated from 2nd degree OM while considering the patient's pain perception. In our study, there has been an increase in opioid administration for $2^{\text {nd }}$ and $3^{\text {rd }}$ transplant procedures.

Management strategy for patients who have transplant indication recommends eradication of any acute or chronic infections before initiating the procedure. Oral antimicrobial prophylaxis is recommended in aplasia after chemotherapy. The blood cultures, taken both from peripheral vein and $\mathrm{CVC}$, were positive for $23 \%$ and $20 \%$ of patients from the two study groups, with predominance of coagulazo-negative staphylococcus. In contrast, digestive colonization were reported in $73.3 \%$ of patients who performed multiple HSCT, showing higher frequency of gram-negative bacteria colonization (Enterobacter-Klebsiella, Pseudomonas, Stenotrophomonas maltophilia, Escherichia coli) or microorganisms associations. Our study shows the correlation between the advanced degree of OM, early onset $(p<0.001)$ and its prolonged duration at the 2 nd and $3 \mathrm{rd}$ transplant procedure. 
Consequently, they require increasing number of hospital admission days, escalated intravenous antibiotic therapy, with impact on both patient's life quality and hospitalization expenses.

The analysis on our 2 study groups does not detect significant differences in terms of neutrophil engraftment, but it reveals the delay of platelet grafting in patients who have performed multiple HSCT procedures $(p=0.029)$, prolonging hospitalization. Literature studies mention the influence of enteral nutrition on early engraftment in these patients $[30,31]$.

Multiple risk factors for the development of severe OM, such as type and tumor stage, cumulative doses of chemotherapy, patient age, oral health and hygiene or pre-transplant nutritional status are identified. In this study there are no correlations found between OM severity and patient age or sex. In contrast, the OM degree was significantly more severe in patients undergoing 2 nd or 3rd transplant procedure, while influence of conditioning regimens was difficult to assess since 54 patients received melphalanbased conditioning. However, OM degree does not influence survival of patients with solid tumors performing HSCT, as they achieved complete remission

\section{REFERENCES}

1. Fuji S, Mori T, Khattry N, Cheng J, Do YR, Yakushijin K, Kohashi S, Fukuda T, Kim SW. Severe weight loss in 3 months after allogeneic hematopoietic SCT was associated with an increased risk of subsequent non-relapse mortality. Bone Marrow Transplant. 2015 Jan;50(1):100-5.

2. Fuji S, Einsele H, Savani BN, Kapp M. Systematic Nutritional Support in Allogeneic Hematopoietic Stem Cell Transplant Recipients. Biol Blood Marrow Transplant. 2015 Oct;21(10):1707-13.

3. Harris DJ, Eilers J, Harriman A, Cashavelly BJ, Maxwell C. Putting evidence into practice: evidence-based interventions for the management of oral mucositis. Clin J Oncol Nurs. 2008 Feb;12(1):141-52.

4. Chaveli-López B. Oral toxicity produced by chemotherapy: A systematic review. J Clin Exp Dent. 2014 Feb 1;6(1):e81-90.

5. Bowen JM, Wardill HR. Advances in the understanding and management of mucositis during stem cell transplantation. Curr Opin Support Palliat Care. 2017 Dec;11(4):341-346.

6. Tunkel AR, Sepkowitz KA. Infections caused by viridans streptococci in patients with neutropenia. Clin Infect Dis. 2002 Jun 1; 34(11):1524-9.

7. Torres P, Díaz J, Arce M, Silva P, Mendoza P, Lois P, Molina-Berríos A, Owen GI, Palma V, Torres VA. The salivary peptide histatin-1 promotes endothelial cell adhesion, migration, and angiogenesis. FASEB J. 2017 Nov;31(11):4946-4958.

8. Yamagata K, Arai C, Sasaki H, Takeuchi Y, Onizawa K, Yanagawa T, Ishibashi N, Karube R, Shinozuka K, Hasegawa Y, Chiba S, Bukawa $\mathrm{H}$. The effect of oral management on the severity of oral mucositis during hematopoietic SCT. Bone Marrow Transplant. 2012 May;47(5):725-30.

9. Escoda-Francolí J, Rodríguez-Rodríguez A, Pérez-García S, Gargallo-Albiol J, Gay-Escoda C. Dental implications in oral cancer patients. Med Oral Patol Oral Cir Bucal. 2011 Jul 1;16(4):e508-13.

10. Chaveli-López B. Oral toxicity produced by chemotherapy: A systematic review. J Clin Exp Dent. 2014 Feb 1;6(1):e81-90. of $\mathrm{OM}$ at the time of discharge from hospital. As confirmed in our study, despite all complications, performing multiple transplant procedures offers higher survival rate for patients with solid tumors.

\section{CONCLUSIONS}

HSCT allows administration of high doses of chemotherapy, new and active additional drugs leading to increased survival rate. Nevertheless, having multiple procedures increase OM severity, negatively influencing clinical evolution and engraftment.

Although OM pathogenesis is analyzed and understood, there are few studies regarding its management in pediatric patients after transplantation. Currently, treatment of OM remains focused on prevention (rigorous oral hygiene, specific measures during chemotherapy and topical products). There is no consensus regarding the recommended type of nutrition, enteral versus parenteral, the choice remaining the clinician's responsibility.

In conclusion, identifying effective measures, encouraging use of modern strategies and developing evidence-based guidelines must be prioritized.

Conflict of interest: none declared Financial support: none declared

11. Sonis ST. Oral mucositis in cancer therapy. J Support Oncol. 2004 Nov-Dec;2(6 Suppl 3):3-8.

12. World Health Organization. Vol. 48. WHO Offset Publication; 1979:15-22

13. Ward EJ, Henry LM, Friend AJ, Wilkins S, Phillips RS. Nutritional support in children and young people with cancer undergoing chemotherapy. Cochrane Database Syst Rev. 2015 Aug 24;(8):CD003298.

14. Banava S, Houshyari M, Safaie T. The effect of casein phosphopeptide amorphous calcium phosphate fluoride paste (CPP-ACPF) on oral and salivary conditions of patients undergoing chemotherapy: A randomized controlled clinical trial. Electron Physician. 2015 Nov 20;7(7):1535-41.

15. Ariyawardana A, Cheng KKF, Kandwal A, Tilly V, et al.; Mucositis Study Group of the Multinational Association of Supportive Care in Cancer/International Society for Oral Oncology (MASCC/ISOO). Systematic review of anti-inflammatory agents for the management of oral mucositis in cancer patients and clinical practice guidelines. Support Care Cancer. 2019 Oct;27(10):3985-3995.

16. Bhatt N, Naithani R, Gupta SK. Supersaturated Calcium Phosphate Rinse in Prevention and Treatment of Mucositis in Patients Undergoing Hematopoietic Stem Cell Transplant. Exp Clin Transplant. 2017 Oct;15(5):567-570.

17. Lu Y, Zhu X, Ma Q, Wang J, Jiang P, Teng S, Zhou L, Wu D, Wang H. Oral cryotherapy for oral mucositis management in patients receiving allogeneic hematopoietic stem cell transplantation: a prospective randomized study. Support Care Cancer. 2020 Apr;28(4):1747-1754.

18. Kamsvåg T, Svanberg A, Legert KG, Arvidson J, von Essen L, Mellgren K, Toporski J, Winiarski J, Ljungman G. Prevention of oral mucositis with cryotherapy in children undergoing hematopoietic stem cell transplantations-a feasibility study and randomized controlled trial. Support Care Cancer. 2020 Oct;28(10):4869-4879.

19. Sung L, Robinson P, Treister N, Baggott T, Gibson P, Tissing W, Wiernikowski J, Brinklow J, Dupuis LL. Guideline for the prevention 
of oral and oropharyngeal mucositis in children receiving treatment for cancer or undergoing haematopoietic stem cell transplantation. BMJ Support Palliat Care. 2017 Mar;7(1):7-16.

20. Raber-Durlacher JE, von Bültzingslöwen I, Logan RM, Bowen J, et al.; Mucositis Study Group of the Multinational Association of Supportive Care in Cancer/International Society of Oral Oncology (MASCC/ISOO). Systematic review of cytokines and growth factors for the management of oral mucositis in cancer patients. Support Care Cancer. 2013 Jan;21(1):343-55.

21. Arends J, Bachmann P, Baracos V, Barthelemy N, Bertz H, Bozzetti $\mathrm{F}$, et al. ESPEN guidelines on nutrition in cancer patients. Clin Nutr. 2017 Feb;36(1):11-48.

22. Seres DS, Valcarcel M, Guillaume A. Advantages of enteral nutrition over parenteral nutrition. Therap Adv Gastroenterol. 2013 Mar;6(2):157-67.

23. Casaer MP, Mesotten D, Hermans G, Wouters PJ, Schetz M, et al. Early versus late parenteral nutrition in critically ill adults. $N$ Engl $J$ Med. 2011 Aug 11;365(6):506-17.

24. Chen Y, Zhao Y, Cheng Q, Wu D, Liu H. The Role of Intestinal Microbiota in Acute Graft-versus-Host Disease. J Immunol Res. 2015;2015:145859.

25. Kuiken NSS, Rings EHHM, van den Heuvel-Eibrink MM, van de Wetering MD, Tissing WJE. Feeding strategies in pediatric cancer patients with gastrointestinal mucositis: a multicenter prospective observational study and international survey. Support Care Cancer. 2017 Oct;25(10):3075-3083.
26. Azarnoush S, Bruno B, Beghin L, Guimber D, Nelken B, YakoubAgha I, Seguy D. Enteral nutrition: a first option for nutritional support of children following allo-SCT? Bone Marrow Transplant. 2012 Sep;47(9):1191-5.

27. Evans JC, Hirani SP, Needle JJ. Nutritional and Post-Transplantation Outcomes of Enteral versus Parenteral Nutrition in Pediatric Hematopoietic Stem Cell Transplantation: A Systematic Review of Randomized and Nonrandomized Studies. Biol Blood Marrow Transplant. 2019 Aug;25(8):e252-e259.

28. Papadopoulou A, Nathavitharana K, Williams MD, et al Diagnosis and clinical associations of zinc depletion following bone marrow transplantation. Archives of Disease in Childhood 1996;74:328-331.

29. Papadopoulou A, Lloyd DR, Williams MD, Darbyshire PJ, Booth IW. Gastrointestinal and nutritional sequelae of bone marrow transplantation. Arch Dis Child. 1996 Sep;75(3):208-13.

30. Gonzales F, Bruno B, Alarcón Fuentes M, De Berranger E, Guimber $D$, et al. Better early outcome with enteral rather than parenteral nutrition in children undergoing MAC allo-SCT. Clin Nutr. 2018 Dec;37(6 Pt A):2113-2121.

31. Azarnoush S, Bruno B, Beghin L, Guimber D, Nelken B, YakoubAgha I, Seguy D. Enteral nutrition: a first option for nutritional support of children following allo-SCT? Bone Marrow Transplant. 2012 Sep;47(9):1191-5. 